\title{
ESTUDO DA DECOMPOSIÇÃO TÉRMICA DE PROPELENTE SÓLIDO COMPÓSITO DE BAIXA EMISSÃO DE FUMAÇA
}

Jony Andrade*, Antonio Gonçalves Frutuoso, Koshun Iha, José Atílio Fritz Fidel Rocco e Eduardo M. Bezerra

Instituto Tecnológico de Aeronáutica, Praça Marechal Eduardo Gomes, 50, 12228-900 São José dos Campos - SP, Brasil

Jivaldo do Rosário Matos

Instituto de Química, Universidade de São Paulo, Av. Prof. Lineu Prestes, 748, 05508-900 São Paulo - SP, Brasil

Maria Encarnación Vázquez Suárez-Iha

Instituto de Química, Universidade de São Paulo Av. Prof. Lineu Prestes, 748, 05508-900 São Paulo - SP / Centro de Ciências

e Humanidades, Universidade Presbiteriana Mackenzie, Rua da Consolação, 930 São Paulo - SP, Brasil

Recebido em 15/2/07; aceito em 17/8/07; publicado na web em 19/12/07

\begin{abstract}
STUDY OF THERMAL DECOMPOSITION OF A SMOKELESS PROPELLANT. The thermal decomposition of hydroxylterminated polybutadiene (HTPB)/ammonium nitrate (AN) based propellants, so called smokeless formulations, and raw materials were investigated by differential scanning calorimetry (DSC) and thermogravimetry (TG). The thermoanalytical profile of different components and of propellant were evaluated and the Arrhenius parameters for the thermal decomposition of the propellant sample were determined by the Ozawa method. The kinetic parameters of the thermal decomposition of propellant samples were determined by DSC measurements. The values obtained for activation energy $\left(\mathrm{E}_{\mathrm{a}}\right)$ and pre-exponential factor were $163 \mathrm{~kJ} \mathrm{~mol}^{-1}$ and $1.94 \times 10^{6}$ $\min ^{-1}$.
\end{abstract}

Keywords: thermal analysis; smokeless; solid propellants.

\section{INTRODUÇÃO}

Sistemas de propulsão aeroespacial que utilizam propelente sólido do tipo compósito ${ }^{1}$ tendem a gerar compostos clorados e alumina, como resultado de seu processo de queima na câmara de combustão do motor-foguete. Estes compostos, quando liberados ao meio ambiente, tendem a deixar um rastro de fumaça que pode ser detectada por técnicas como infravermelho (IR) denunciando, assim, a posição de lançamento do artefato além de sua trajetória ${ }^{2}$.

Conseqüentemente, a fração de alumínio metálico empregado nas formulações de propelentes compósitos do tipo "smokeless" não deve ser superior a $2 \%$ em massa. Entretanto, esta limitação no percentual de alumínio ocasiona o surgimento de fenômenos de instabilidade de queima, além de uma diminuição do impulso específico (Isp), principal parâmetro balístico para formulações de propelentes. Em razão disso, tem-se uma busca constante por novos compostos e formulações ${ }^{3}$.

As técnicas termoanalíticas, entre outras, podem contribuir no estudo de novos materiais e formulações para utilização em sistemas de propulsão ${ }^{4}$, gerando informações quanto às propriedades de decomposição térmica do material, como, por exemplo, seu comportamento em queima e os fenômenos de transição de fases cristalinas dos sais oxidantes observados durante o processo de combustão ${ }^{5}$.

Neste trabalho é apresentado um estudo sobre a decomposição térmica de um propelente sólido baseado no polibutadieno líquido hidroxilado (PBLH), nitrato de amônio (AN) e outros componentes, utilizando-se a calorimetria exploratória diferencial (DSC) e a termogravimetria (TG). Este estudo visou avaliar os fenômenos que ocorrem durante a queima, para obter uma orientação em relação ao potencial de aplicação da formulação do compósito considerado. Foram gerados, ainda, os parâmetros cinéticos de decomposição térmica da formulação de propelente adotada, utilizandose o modelo cinético de Flynn, Wall e Ozawa ${ }^{6}$.

*e-mail: jonyquim@ita.br

\section{Modelo cinético de Flynn, Wall e Ozawa}

O estudo da cinética de decomposição térmica de materiais no estado sólido tem como conceito que a variação da massa do composto, em decorrência de um estímulo térmico, em função do tempo, obedece a seguinte equação

$\frac{d \alpha}{d t}=k(T) f(\alpha)$

onde " $\alpha$ " representa a fração de conversão da amostra, " $t$ " o tempo para a conversão, "T" a temperatura absoluta, "k(T)" a constante cinética em função da temperatura e " $f(\alpha)$ " a função que representa o modelo de decomposição térmica para o processo em estudo.

A constante cinética de uma reação obedece à Equação de Arrhenius

$k(T)=A e^{-E_{a} / R T}$

onde "E " é a energia de ativação, "A" o fator pré-exponencial, "R" a constante universal dos gases e "T" a temperatura absoluta. Substituindo-se a Equação 2 em 1, tem-se:

$\frac{d \alpha}{d t}=A e^{-E_{a} / R T} f(\alpha)$

Integrando-se a Equação 3, obtém-se:

$\int_{0}^{\alpha} \frac{d \alpha}{f(\alpha)}=\int_{0}^{t} A e^{-E_{a} / R T} d t=g(\alpha)$

Em processos não-isotérmicos, onde um sólido é submetido a uma razão de aquecimento $(\beta), \beta=d T / d t$, e substituindo-se esta expressão na Equação 4, obtém-se então: 
$\int_{0}^{\alpha} \frac{d \alpha}{f(\alpha)}=\frac{A}{\beta} \int_{T_{0}}^{T} e^{-E_{a} / R T} d T=g(\alpha)$

Resolvendo-se a integral da Equação 5, e com a utilização da aproximação de Doyle ${ }^{7}$, chega-se à expressão:

$\log \beta=-0,457 \frac{E_{a}}{R T}-2,315+\log \left(\frac{A E_{a}}{R}\right)-\log (g(\alpha))$

Portanto, para um processo onde uma amostra é submetida a aquecimento controlado, sob uma determinada razão de aquecimento $\beta$, a inclinação da reta obtida para $\log \beta$ vs $1 / \mathrm{T}$ é igual a $-0,457 \mathrm{E}_{\mathrm{a}} /$ $\mathrm{R}$, onde $\mathrm{T}$ é a temperatura relativa à altura máxima da curva termoanalítica, também conhecida como temperatura de pico $\left(\mathrm{T}_{\mathrm{m}}\right)$. A partir desta definição, tem-se que a energia de ativação pode ser calculada a partir da seguinte equação

$$
E_{a}=-2,19 R\left(\frac{d \log \beta}{d\left(1 / T_{m}\right)}\right)
$$

Considerando um modelo que assume uma reação de $1^{\text {a }}$ ordem, pode-se calcular o fator pré-exponencial "A", integrando-se a Equação 5. A seguinte equação é gerada:

$$
A=\frac{1}{R T_{m}^{2}} \beta E_{a} e^{E_{a} / R T_{m}}
$$

Assim, obtendo-se as respectivas curvas termoanalíticas em diferentes razões de aquecimento, determina-se a energia de ativação $\left(\mathrm{E}_{\mathrm{a}}\right)$ do processo construindo-se o gráfico do logaritmo da razão de aquecimento $(\beta)$ em função do inverso da temperatura de pico $\left(1 / \mathrm{T}_{\mathrm{m}}\right)$ e o fator pré-exponencial (A) pode ser então determinado a partir da Equação 8. Este procedimento pode ser empregado tanto para curvas obtidas pela termogravimetria (TG), quanto para as obtidas por calorimetria exploratória diferencial (DSC) ${ }^{8}$.

\section{PARTE EXPERIMENTAL}

\section{Procedimento de preparo do grão propelente}

O propelente sólido compósito foi obtido misturando-se $75 \%$ $(\mathrm{m} / \mathrm{m})$ de nitrato de amônio (AN) da marca Merck S/A com $22 \%$ $(\mathrm{m} / \mathrm{m})$ de PBLH e $1 \%(\mathrm{~m} / \mathrm{m})$ do plastificante dioctilftalato (DOP), sob agitação constante e moderada. A mistura obtida foi levada para desaeração em um sistema a pressão reduzida $(20 \mathrm{~mm} \mathrm{Hg})$, sob uma temperatura de $60{ }^{\circ} \mathrm{C}$. Nesta etapa, a determinação da viscosidade da massa de propelente apresentou um valor de $200.000 \mathrm{cPs}$. Em seguida, foram adicionados $2 \%(\mathrm{~m} / \mathrm{m})$ de alumínio metálico completando o percentual total da massa de propelente.

O processo de cura (extensão da cadeia polimérica) do propelente consistiu na adição do diisocianato de isoforona (IPDI), com teor de NCO livre de $8,95 \mathrm{meq} \mathrm{NCO} / \mathrm{g}$, de modo que a relação NCO/OH, que é a relação entre o poliol (PBLH) e o diisocianato (IPDI), apresentou um valor igual a 0,93. Após a adição do agente de cura (IPDI), a mistura foi colocada sob agitação por 20 min e, em seguida, carregada nos corpos de prova. Estes corpos de prova foram levados para estufa para um processo de cura por 5 dias a $65{ }^{\circ} \mathrm{C}$. Ao término do ciclo de cura, os corpos de prova foram acondicionados em um dessecador para evitar a absorção de umidade pelo nitrato de amônio, garantindo a integridade dos ensaios realizados posteriormente.

\section{Estudo termoanalítico}

As curvas TG/DTG e DSC foram obtidas sob atmosfera dinâmica de $\mathrm{N}_{2}\left(50 \mathrm{~mL} \mathrm{~min}^{-1}\right)$, utilizando uma razão de aquecimento de $10{ }^{\circ} \mathrm{C}$ $\min ^{-1}$, tanto para amostras de matérias-primas quanto para o propelente sólido. A determinação dos parâmetros cinéticos de decomposição térmica da formulação de propelente foi realizada mediante célula calorimétrica DSC-50 da marca Shimadzu nas razões de aquecimento de $10 ; 12,5 ; 17,5$ e $20{ }^{\circ} \mathrm{C} \mathrm{min}^{-1}$, empregando-se cadinho de alumínio parcialmente fechado, contendo massa de amostra de cerca de $2 \mathrm{mg}$. As curvas TG/DTG foram obtidas a partir da termobalança modelo TGA 50 da marca Shimadzu, empregando cadinhos de platina e massa de amostra de aproximadamente $2 \mathrm{mg}$.

\section{RESULTADOS E DISCUSSÃO}

\section{Avaliação termoanalítica do polibutadieno líquido hidroxilado}

Inicialmente, a matéria-prima estudada foi o polibutadieno líquido hidroxilado (PBLH), sob as condições descritas anteriormente, obtendo-se as respectivas curvas DSC e TG/DTG, apresentadas na Figura 1. As curvas TG/DTG do PBLH mostraram que o evento principal de decomposição do poliol situa-se na faixa de 200 a $510{ }^{\circ} \mathrm{C}$, com perda de massa de aproximadamente $91 \%$. Nesta faixa de temperatura, as curvas DSC apresentaram dois eventos térmicos, um exotérmico $\left(380{ }^{\circ} \mathrm{C}\right)$ e outro endotérmico $\left(450{ }^{\circ} \mathrm{C}\right)$. O evento exotérmico está relacionado a processos de "crosslinking" da cadeia polimérica ("binder"), despolimerização e ciclização com decomposição térmica ${ }^{9}$. O evento de decomposição térmica é exotérmico e prevalece sobre os outros que, caracteristicamente, são endotérmicos.
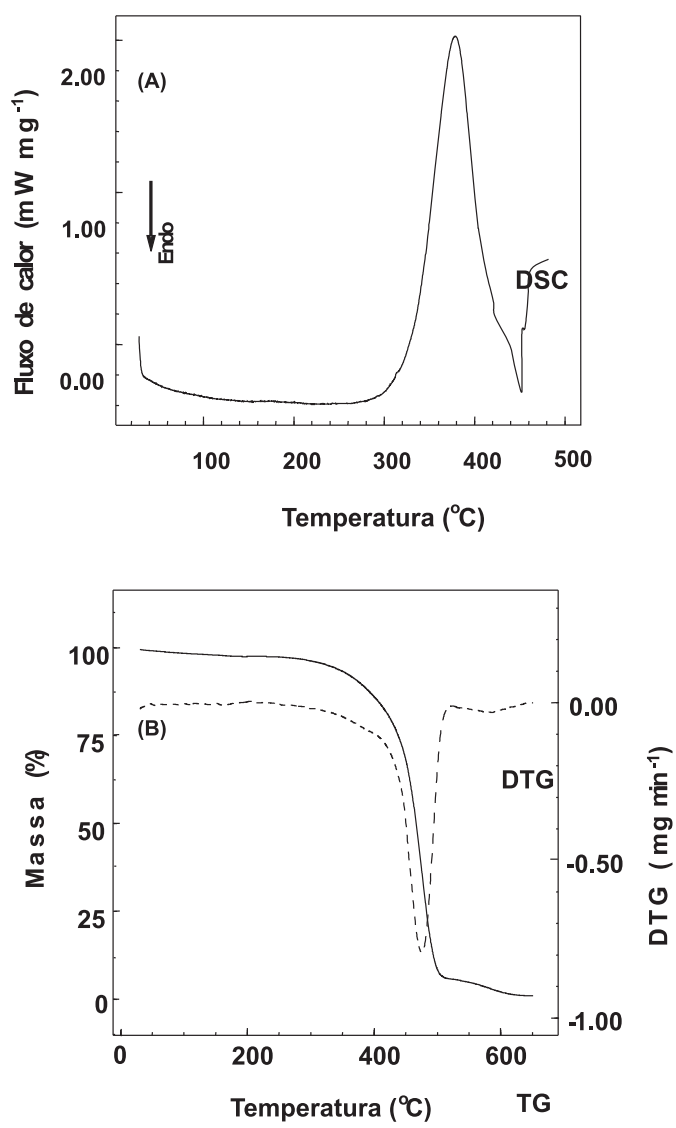

Figura 1.Curvas DSC (A) e TG/DTG (B) obtidas a $10^{\circ} \mathrm{C} \mathrm{min}^{-1}$ e sob atmosfera dinâmica de $\mathrm{N}_{2}\left(50 \mathrm{~mL} \mathrm{\textrm {min } ^ { - 1 }}\right)$ para a amostra de $\mathrm{PBLH}$ 
A variação da entalpia obtida para o evento exotérmico resultou em um valor de $886 \mathrm{~J} \mathrm{~g}^{-1}$. Este valor indica que a energia envolvida no processo de decomposição térmica é superior à energia envolvida no rompimento das ligações estabelecidas no processo de polimerização do PBLH. O evento endotérmico, observado próximo de $450^{\circ} \mathrm{C}$, refere-se ao consumo de energia necessário à eliminação dos produtos gasosos gerados na primeira fase. Em termos de perda de massa, as curvas TG/DTG indicam que a decomposição térmica é praticamente completa até $650{ }^{\circ} \mathrm{C}$, restando um resíduo de cerca de $1 \%$ em relação à massa inicial.

\section{Avaliação termoanalítica do nitrato de amônio (AN)}

As curvas TG/DTG e DSC obtidas para o nitrato de amônio são apresentadas na Figura 2. A curva DSC obtida para esta espécie química apresenta quatro eventos endotérmicos na faixa de temperatura entre 50 e $300{ }^{\circ} \mathrm{C}$. O primeiro evento endotérmico está relacionado à mudança de fase cristalina do composto de ortorrômbico $\beta$ (IV) para ortorrômbico $v$ (III) com temperatura de pico observada em $52^{\circ} \mathrm{C}$. Esta mudança da fase cristalina é acompanhada pelo aumento de volume do cristal $^{10}$, o qual representa um grave problema de estabilidade dimensional do grão propelente sólido. Esta alteração pode produzir descontinuidades na massa alterando suas características de queima, levando, assim, a um súbito aumento da frente da chama e a uma provável explosão do motor-foguete.
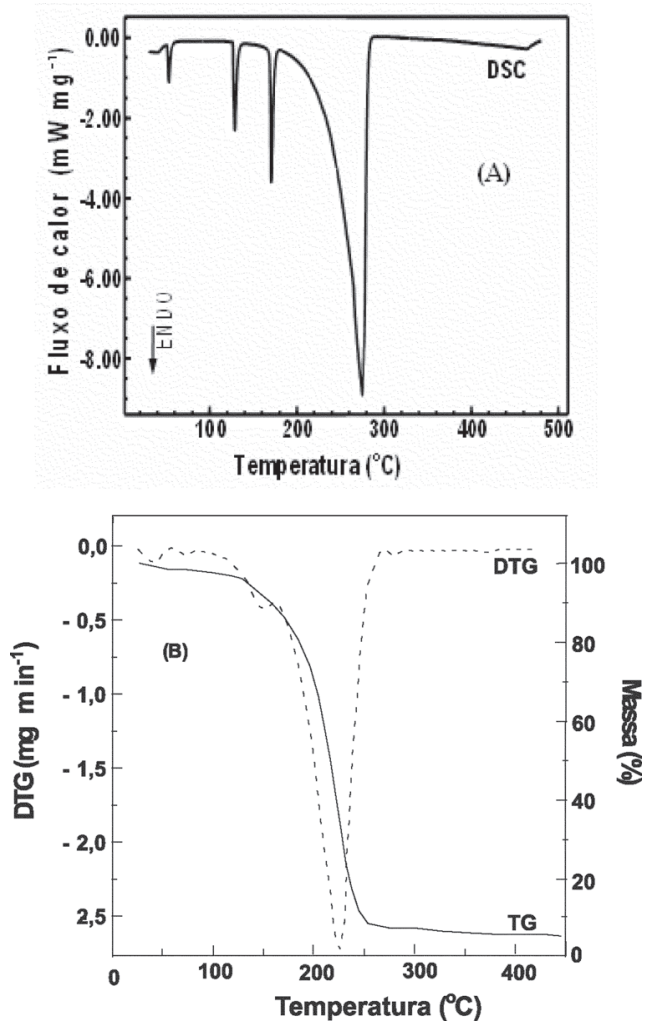

Figura 2. Curvas DSC (A) e TG/DTG (B) obtidas a $10^{\circ} \mathrm{C} \mathrm{min}^{-1}$ e sob atmosfera dinâmica de $N_{2}\left(50 \mathrm{~mL} \mathrm{~min}^{-1}\right)$ para a amostra de nitrato de amônio

A temperatura de pico observada por volta de $128{ }^{\circ} \mathrm{C}$ está relacionada à mudança da estrutura cristalina do nitrato de amônio de ortorrômbica para tetragonal, e de tetragonal para cúbica na temperatura de $170{ }^{\circ} \mathrm{C}$, onde não ocorre variação de volume. Em relação ao evento principal de decomposição térmica a curva DTG evidenciou uma temperatura de pico em $227^{\circ} \mathrm{C}$, porém, na curva DSC encontrou-se uma temperatura de pico em $275^{\circ} \mathrm{C}$.
Em uma análise da curva TG foi observado que a decomposição térmica da amostra termina próximo a $350{ }^{\circ} \mathrm{C}$ com $95 \%$ de perda em relação à massa inicial. Observa-se, portanto, um percentual de resíduo de cerca de 5\% que, a princípio, pode ser atribuído à presença de impurezas. Entretanto, é provável que o mesmo seja relacionado ao produto de decomposição do nitrato de sódio residual do processo de síntese do nitrato de amônio ${ }^{11}$, uma vez que nitrato de sódio é um dos reagentes primários utilizados na sua obtenção.

Os dados obtidos para a avaliação termoanalítica do nitrato de amônio estão em acordo com os apresentados por Tompa ${ }^{12}$, que registrou os três eventos de transição da fase cristalina do nitrato de amônio nas temperaturas de 54,129 e $170{ }^{\circ} \mathrm{C}$ e o evento principal relacionado à perda de massa (decomposição) a $269^{\circ} \mathrm{C}$. As variações observadas entre as temperaturas registradas por Tompa ${ }^{12} \mathrm{e}$ as obtidas neste trabalho devem-se às razões de aquecimento adotadas, uma vez que Tompa ${ }^{12}$ adotou uma razão de $5{ }^{\circ} \mathrm{C} \min ^{-1}$.

\section{Avaliação termoanalítica do propelente (PBLH: AN: DOP)}

O estudo termoanalítico da amostra de propelente foi iniciado conforme descrito na parte experimental, obtendo-se as curvas TG/ DTG e DSC nas mesmas condições em que foram realizados os ensaios das respectivas matérias-primas. As curvas TG/DTG e DSC (Figura 3) evidenciam a presença do nitrato de amônio (AN) na formulação do propelente compósito. Isso é confirmado pelo registro dos 3 picos endotérmicos $\left(52,128\right.$ e $\left.170{ }^{\circ} \mathrm{C}\right)$ característicos das mudanças de fases cristalinas, ocorridas para o $\mathrm{AN}$, os quais são concordantes com os observados na curva DSC da amostra estudada separadamente. Na curva DSC, Figura 2A, o primeiro evento térmico correspondente à transição cristalina do $\mathrm{AN}\left(52{ }^{\circ} \mathrm{C}\right)$ está sobreposto pelo evento endotérmico característico da eliminação de água, que ocorre entre 25 e $110^{\circ} \mathrm{C}$.

Da observação das curvas TG/DTG (Figura 3B), nota-se que a decomposição térmica da amostra da formulação de "smokeless" ocorre em três etapas principais: a primeira, compreendida entre 25 e $120^{\circ} \mathrm{C}$, que pode ser atribuída à presença de água não ligada (umidade), representando $14 \%$ da massa inicial da amostra. Cabe salientar, entretanto, que esta umidade não provoca o surgimento de fumaça, devido à alta temperatura do sistema em combustão. A segunda etapa, observada entre 120 e $320^{\circ} \mathrm{C}$, representa uma perda de massa de aproximadamente $50 \%$ e está relacionada à decomposição térmica da maior parte do sal oxidante (AN), da parcela do poliol (PBLH). A terceira etapa de decomposição térmica da amostra, entre 320 e $540{ }^{\circ} \mathrm{C}$, representa a decomposição térmica da porção principal do $\mathrm{PBLH}$, não decomposta na etapa anterior e que corresponde a $24 \%$ de perda da massa inicial da amostra. A temperatura de pico na curva DSC da amostra, representando o evento principal de decomposição térmica do material, está localizada em $255^{\circ} \mathrm{C}$, com uma variação de entalpia $(\Delta \mathrm{H})$ da ordem de $217 \mathrm{~J} \mathrm{~g}^{-1}$.

Estes resultados mostram alguns dos problemas que envolvem a utilização do nitrato de amônio como oxidante nas formulações de "smokeless". Um dos problemas pode ser associado à alta porcentagem de água presente no material, cerca de $14 \%$ da massa inicial da amostra do compósito (PBLH/NA). O fato do nitrato de amônio ser altamente higroscópico representa um problema adicional em termos de estocagem, para sistemas de propulsão aeroespaciais que se baseiam nestas formulações. Isto impõe que o acondicionamento destes motores deve ser feito em ambientes isentos de umidade. Além disto, exige um pré-tratamento do AN antes de ser incorporado ao poliol, uma vez que esta umidade pode comprometer a reação do PBLH com o diisocianato de isoforona, IPDI, devido à priorização da reação entre a água e o isocianato. Esta reação resulta na formação de $\mathrm{CO}_{2}$ que tende a produzir des-conti- 

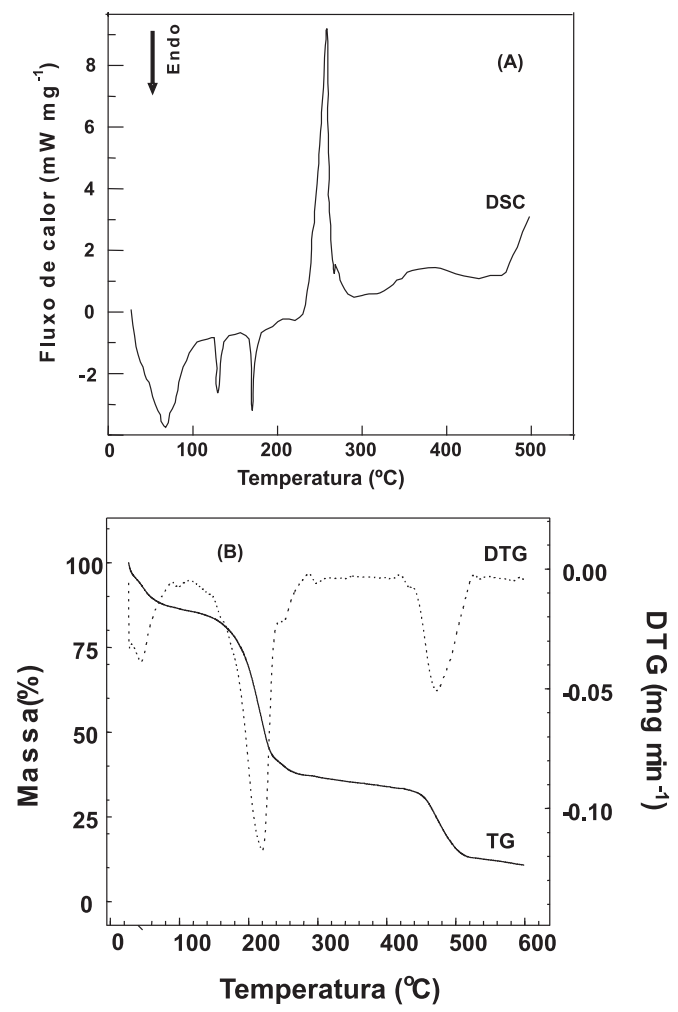

Figura 3. Curvas DSC (A) e TG/DTG (B) obtidas a $10^{\circ} \mathrm{Cmin}^{-1}$ e sob atmosfera dinâmica de $N_{2}\left(50 \mathrm{~mL} \mathrm{~min}^{-1}\right)$ para a amostra de propelente sólido

nuidades (bolhas) no grão propelente sólido.

Uma possível solução para este problema pode ser obtida com o emprego de "binders ativos" em substituição ao PBLH, como os baseados nos polímeros derivados de metil-azotetos de glicidilas, possibilitando a utilização do nitrato de amônio como oxidante. Isto porque a perda de impulso específico (Isp) pode ser compensada pela atividade do "binder", ou então, pelo uso de um nitrato de amônio modificado, conhecido como PSAN-"Phase-stabilized ammonium nitrate". O PSAN foi desenvolvido para se evitar a transição da fase cristalina de ortorrômbica IV $\rightarrow$ III, evitando o efeito indesejável da variação de volume do $\operatorname{cristal}^{10}$.

Por fim, aplicando-se o método cinético de Ozawa para a formulação de "smokeless" composta do PBLH e AN, sob diferentes razões de aquecimento, foram obtidas as curvas DSC apresentadas na Figura 4. O tratamento dos dados resultou no gráfico de Ozawa, Figura 5.

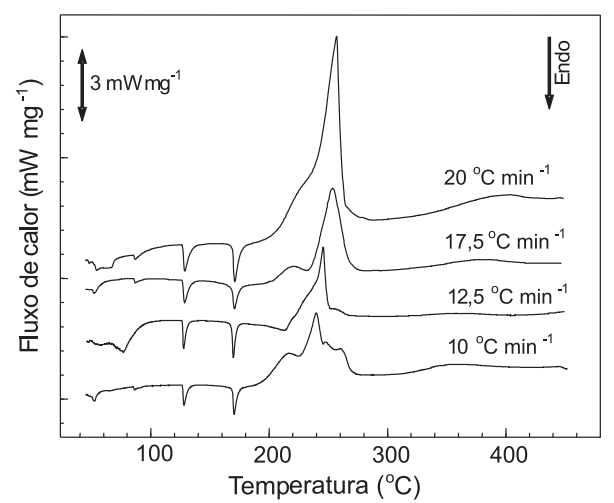

Figura 4. Curvas DSC obtidas sob diferentes razões de aquecimento para a formulação de propelente à base de PBLH e AN

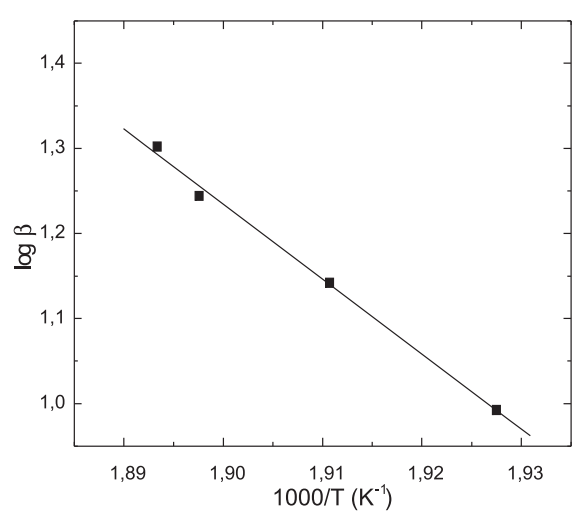

Figura 5. Gráfico de Ozawa obtido a partir das curvas DSC da figura 4

Observa-se na Figura 4 a ocorrência do estreitamento das curvas à medida que ocorre o aumento da razão de aquecimento. Isto se deve à diminuição do tempo necessário para que a amostra alcance uma determinada temperatura. As curvas DSC foram consideradas para cálculo dos parâmetros cinéticos em virtude do método ter como referência a temperatura de pico, ou seja, a temperatura referente ao ponto mais alto da curva. Em relação às respectivas curvas DSC obtidas para o propelente, tem-se que o evento principal do processo se refere ao pico exotérmico. Cabe salientar que o método de deslocamento do pico de Ozawa é a base da norma ASTM 698-05, sendo muito utilizada para estudos cinéticos de materiais energéticos ${ }^{6}$.

Finalmente, utilizando-se o software da Shimadzu disponível no sistema termoanalítico gerou-se o gráfico de Ozawa (Figura 5) e os parâmetros cinéticos foram calculados, como $\mathrm{E}_{\mathrm{a}}=163 \mathrm{~kJ} \mathrm{~mol}^{-1} \mathrm{e}$ $\mathrm{A}=1,94 \times 10^{16} \mathrm{~min}^{-1}$. O bom ajuste dos 4 pontos à reta apresentada no gráfico de Ozawa é um indício de que o método cinético adotado foi adequado e pode ser utilizado na análise de compósitos como as formulações de "smokeless" do presente estudo.

\section{CONCLUSÃO}

As transições de fases cristalinas observadas nas curvas termoanalíticas obtidas para o nitrato de amônio também foram observadas nas curvas termoanalíticas da amostra de propelente compósito acarretando, assim, a atribuição de desvantagens ao propelente quanto a um alto percentual de umidade e, ainda, possíveis efeitos de instabilidades de queima. Pode-se destacar que a formulação de propelente promove uma baixa liberação de energia em relação, por exemplo, às formulações tradicionais de propelentes sólidos, como o representado pelo polibutadieno líquido hidroxilado (PBLH) carregado com perclorato de amônio ${ }^{13}$ (AP), que chega a $497 \mathrm{~J} \mathrm{~g}^{-1}$. Por fim, o modelo cinético de Flynn, Wall e Ozawa ${ }^{7}$ apresentou boa aplicabilidade para obtenção dos parâmetros cinéticos de decomposição térmica da formulação de propelente abordada no presente trabalho.

\section{DEDICATÓRIA}

O presente trabalho é dedicado ao Prof. Dr. Eduardo Fausto de Almeida Neves ${ }^{14}$.

\section{AGRADECIMENTOS}

Ao CNPq, à FAPESP e CAPES pelo apoio financeiro. 


\section{REFERÊNCIAS}

1. Sciamareli, J.; Takashi, K. F. M.; Teixeira, J. M.; Iha, K.; Quim. Nova 2002, 25, 107.

2. Folly, P.; Mader, P.; Chimia 2004, 58, 374.

3. Laredo, D.; Netzer, W. D.; J. Quant. Spectrosc. Radiat. Transfer 1993, $50,511$.

4. Paterlini, W. P.; Botelho, E. C.; Rezende, L. C.; Lourenço, V. L.; Rezende, M. C.; Quim. Nova 2002, 25, 221.

5. Falone, S. Z.; Vieira, E. M.; Quim. Nova 2004, 27, 849.

6. Standard Test Method for Arrhenius Kinetic Constants for Thermally Unstable Materials Using Differential Scanning Calorimetry and the Flynn/ Wall/Ozawa Method, 2005, E 698-05.
7. Yeu-Cherng, L.; Kuo, K. K.; Thermochim. Acta 1996, 275, 181.

8. Silva, G.; Mattos, E. C.; Nakamura, N.; Iha, K.; Quim. Nova 2004, $27,889$.

9. Al-Harthi, A.; Willians, A.; Fuel 1998, 77, 1451.

10. Carvalheira, P.; Gadiot, G. M. H. J. L.; Klerk, W. P. C.; Thermochim. Acta 1995, 269, 273.

11. Korošec, R. C.; Kajič, P.; Bukovec, P.; J. Therm. Anal. Calorim. 2007, 89, 1.

12. Tompa, A. S.; Thermochim. Acta 2000, 357, 177.

13. Rocco, J. A. F. F.; Lima, J. E. S.; Frutuoso, A. G.; Iha, K.; Ionashiro, M.; Matos, J. R.; Suarez-Iha, M. E. V.; J. Therm. Anal. Calorim. 2004, 75, 551 Rocco, J. A. F. F.; Lima, J. E. S.; Frutuoso, A. G.; Iha, K.; Ionashiro, M.; Matos, J. R.; Suáres-Iha, M. E. V.; J. Therm. Anal. Calorim. 2004, 77, 803. 14. Gutz, I. G. R.; Quim. Nova 2006, 29, 1150. 